\title{
Changes of fractional anisotropy and RGMa in crossed cerebellar diaschisis induced by middle cerebral artery occlusion
}

\author{
YONG ZHANG, XIAO WANG, JINGLIANG CHENG, YANAN LIN, \\ LU YANG, ZHENGHAO CAO and YUNJUN YANG
}

Department of Radiology, The First Affiliated Hospital of Zhengzhou University, Zhengzhou, Henan 450052, P.R. China

Recieved September 24, 2017; Accepted July 9, 2018

DOI: $10.3892 /$ etm.2019.7986

\begin{abstract}
Crossed cerebellar diaschisis (CCD) is the phenomenon of hypoperfusion and hypometabolism of the contralateral cerebellar hemisphere caused by dysfunction of the associated supratentorial region. The aim of the present study was to analyze the changes in fractional anisotropy (FA) in CCD induced by middle cerebral artery occlusion (MCAO) using magnetic resonance-diffusion tensor imaging (MR-DTI). Furthermore, the role of repulsive guidance molecule a (RGMa) in CCD was assessed by measuring RGMa expression using histochemical analysis. In the present study, the cerebellar hemisphere was serially scanned with T2-weighted, serial diffusion-weighted and diffusion tensor (DT) imaging using a 3.0T GE Signa HDxt Scanner to analyze the changes in FA over $72 \mathrm{~h}$. Subsequently, immunohistochemistry analyses of the corresponding cerebellar hemisphere sections were performed to assess the expression of RGMa. Results indicated that FA of both sides of the cerebellar hemisphere, particularly that of the contralateral cerebellar hemisphere (right side) derived from DTI, was reduced during the 72 -h time period following MCAO, and the decrease was maximal and statistically significant at $12 \mathrm{~h}(\mathrm{P}<0.05)$. Immunohistochemistry analysis revealed a significant increase in the expression of RGMa protein in the affected region of the contralateral cerebellar hemisphere (right side) at $24 \mathrm{~h}$ following MCAO injury $(\mathrm{P}<0.05)$. Furthermore, the expression of RGMa and FA was negatively correlated in MCAO $(\mathrm{P}<0.05)$. The results suggest that MR-DTI is an important assessment to evaluate changes of FA in CCD induced by MCAO. Furthermore, the present results suggest that RGMa, which was negatively correlated with FA in MCAO rats, may serve an important role in CCD.
\end{abstract}

Correspondence to: Dr Jingliang Cheng, Department of Radiology, The First Affiliated Hospital of Zhengzhou University, 1 Jianshe East Road, Erqi, Zhengzhou, Henan 450052, P.R. China

E-mail: chengjl_2016@163.com

Key words: middle cerebral artery occlusion, crossed cerebellar diaschisis, repulsive guidance molecule a, magnetic resonance diffusion tensor imaging

\section{Introduction}

Cerebral infarction is the most common cerebrovascular disease and is associated with high morbidity and mortality rates $(1,2)$. In addition to damaging the site of occurrence, a cerebral infarction also damages distantly located regions of a tissue. For instance, cerebral infarctions may lead to crossed cerebellar diaschisis (CCD) $(3,4)$. CCD following a cerebellar infarction is associated with depressed cerebral metabolism and blood flow in the cerebellar hemisphere located contralateral to the focal supratentorial lesion (5). A disruption of the corticopontocerebellar pathway connecting the infarcted cerebellum and contralateral hemisphere has been identified as the most likely cause of CCD (6). Notably, the diminished excitatory trans-synaptic neuronal input caused by morphological degeneration is considered to account for the subsequent reduction in metabolism and blood flow (7). Baron et al (8) were the first to report CCD in the cerebral hemisphere contralateral to the central region of supratentorial ischemic infarction. Since then, this phenomenon has been observed in various clinical conditions, including intracranial tumors (9), arteriovenous malformations (10), and hemorrhages (11). Previously, positron emission tomography and single-photon emission computed tomography were used to detect CCD $(12,13)$. However, these techniques are expensive and involve the use of radiation. The use of magnetic resonance imaging (MRI) enables the visualization of damage without an exposure to radiation. However, conventional MRI is not sufficiently sensitive for the detection of CCD in its early phase. A more recently developed form of MR, diffusion tensor imaging (DTI), has allowed for the detection of altered white matter fibers. Hence, it has enabled an accurate assessment of various brain disorders (14). The superior spatial resolution of this type of MRI is sensitive in detecting subtle morphologic changes in affected cerebellar hemispheres (15).

Despite the mounting evidence of the association of transneuronal depression with CCD, the mechanism of CCD pathophysiology is still not completely understood (7). The repulsive guidance molecule a(RGMa) has been demonstrated to impede neurite outgrowth in postnatal cerebellar neurons $(16,17)$. In rats, the induction of RGMa expression following spinal cord injury at the site of the lesion has been observed (18). Neutralization of RGMa with local administration of an antibody was identified to significantly facilitate 
axon regeneration following spinal cord injury $(15,19)$. In addition, RGMa has been indicated to participate in the development of scar tissue following injury and in the myelination of fiber tracts (18). Furthermore, RGMa has been indicated to be one of the most potent inhibitors of axonal growth (17). In a previous study, the RGMb expression levels in the brain tissue of rats with MCAO were enhanced and this effect was suggested to be involved in the regeneration and remodeling of axons and synapses after cerebral ischemia injury (20). Furthermore, RGMa suppressed angiogenesis following ischemia and reperfusion injury in a rat MCAO model (21).

In the present study, CCD was induced in rats by occluding the MCA and the relevant changes were detected using MR-DTI. The changes were further quantified by determining the fractional anisotropy (FA). Subsequently, in order to understand the pathophysiology of CCD, the role of RGMa was investigated in this disorder and the expression of RGMa in sections with compromised fiber integrity was also determined using MR-DTI.

\section{Materials and methods}

Animals. A total of 70 adult male Specific Pathogen Free Sprague Dawley rats (age, 10-12 weeks; weight, 270-320 g) were purchased from the Laboratory Animal Center of Hennan Province (Zhengzhou, China) and bred in the Experimental Animal Center of Zheng Zhou University (Zhengzhou, China) with constant temperature $\left(22-25^{\circ} \mathrm{C}\right)$ and humidity $(40-60 \%)$, a 12 h-light/dark cycle and free access to standard chow and water prior to- and post-surgical intervention. The experimental protocols were approved by the Institutional Animal Care and Use Committee of Zhengzhou University (Zhengzhou, China).

Establishment of the MCAO model. Rats were randomly divided into two groups: Sham surgery (sham control, $n=14$ ) and MCAO $(n=56)$. The MCAO rats were randomly divided into a further seven groups $(n=8)$ according to the $h$ assessed following surgery (at 1, 3 6, 9, 12, 24 and $72 \mathrm{~h}$ ). MCAO was induced as previously described by Longa et al (22). Briefly, the rats were anesthetized intraperitoneally with ketamine $(80 \mathrm{mg} / \mathrm{kg})$ and xylazine $(10 \mathrm{mg} / \mathrm{kg})$. Anesthesia was maintained with $1.5 \%$ isoflurane with $0.5-1.0 \mathrm{l} / \mathrm{min} 100 \% \mathrm{O}_{2}$ through a face mask. The common carotid artery, external carotid artery (ECA) and internal carotid artery (ICA) were exposed and a 4-cm long 3-0 monofilament nylon suture (Harvard Apparatus, Cambridge, MA, USA) was used to tie the proximal ECA, ICA and the circle of Willis effectively to occlude the MCA. Following an occlusion period of $60 \mathrm{~min}$, the suture was withdrawn to allow reperfusion. For sham surgery, all arteries were exposed during the surgical period but the filament was not inserted into the MCA. Following surgery, the rats were housed individually and closely monitored for changes in behavior and vital signs. The MCAO model was considered successfully established when the following observations were indicated: i) Horner syndrome occurred in the ipsilateral (left side) when the rat displayed wakefulness after surgery; ii) the forelimbs did not completely stretch; and iii) contralateral circling occurred when walking. Simultaneously, the Zea-Longa neurological deficit scores were calculated. Scores of 2 and 3 were included in the MCAO model. The neurological scores were blindly assessed independently by two pretrained technicians when the rats awoke after MCAO surgery according to the Zea-Longa neurological deficit scores (12). The Zea-Longa assessment criteria were as follows: Score 0, normal, no neurological sign; score 1, cannot completely stretch contralateral forelimbs; score 2, contralateral circling when walking; score 3, contralateral fall over when walking; and score 4, cannot walk and lowering of consciousness.

$M R$. Imaging of the experimental animals was performed at $1,3,6,9,12,24$ and $72 \mathrm{~h}$ and imaging of the sham control animals was performed at $0 \mathrm{~h}$ following surgery using a Signa HDxt 3.0T MR scanner (GE Healthcare, Chicago, IL, USA) employing the multi-channel coil designed for rats (the Medical Science and Technology Corporation of Chenguang, Shanghai, China). Imaging consisted of axial T1-T2-weighted (T2W), sagittal T2W, DWI and DTI sequence. The scanning parameters were as follows: Fast spin-echo (FSE) sequence T1WI, time of repetition $(\mathrm{TR}) / \mathrm{echo}$ time $(\mathrm{TE})=360 / 23.3 \mathrm{msec}$, field of view $(F O V)=70 \times 70 \mathrm{~mm}$, number of excitations $(\mathrm{NEX})=4.00$ and matrix $320 \times 256$; FSE sequence T2WI, TR/TE=2,300/115.3 msec, FOV=110x110 mm, NEX=4 and matrix 256x256; single-shot spin-echo/echo-planar imaging sequence DWI, TR/TE=2350/78.9 msec, FOV=110x110 mm, $\mathrm{NEX}=4.00$ and matrix $96 \times 96$; and DTI, TR $/ \mathrm{TE}=2500 / 92.2 \mathrm{msec}$, $\mathrm{FOV}=110 \times 110 \mathrm{~mm}, \mathrm{NEX}=4.00$ and matrix $128 \times 128$.

Post-imaging processing. MR images were subjected to a series of processing and statistical analyses by the workstation provided by the GE company. Images obtained during DTI were processed using the Workstation software version 5.4.07 (GE Healthcare). The threshold was adjusted by covering the brain tissue with green lines. Apparent diffusion coefficient (ADC) and FA maps were obtained and images were batch saved. Regions of interest, including the infarct core and bilateral cerebellar hemispheres, were measured on FA maps. The measurements of each site were repeated three times and data were recorded as an average.

Immunohistochemistry analysis. Following sacrifice, the cerebellar hemispheres were excised and fixed in phosphate-buffered $4 \%$ formaldehyde for $24 \mathrm{~h}$ at $4^{\circ} \mathrm{C}$, wax-embedded and cut into $5-\mu \mathrm{m}$ sections prior to being transferred to slides. Sections were treated with $3 \% \mathrm{H}_{2} \mathrm{O}_{2}$, blocked with $5 \%$ rabbit serum (Shanghai Haoran Biological Technology Co., Ltd.) for $20 \mathrm{~min}$ at $25^{\circ} \mathrm{C}$, and incubated overnight at $4^{\circ} \mathrm{C}$ with RGMa antibody (1:100; cat. no. ab216643; Abcam, Cambridge, MA, USA). Subsequently, samples were treated with horseradish peroxidase-labeled secondary goat anti-rabbit IgG antibody (1:10,000; cat. no. 7074; Cell Signaling Technology, Inc., Danvers, MA, USA) for $30 \mathrm{~min}$ at $37^{\circ} \mathrm{C}$, stained with 3,3-'diaminobenzidine for $3 \mathrm{~min}$ at $25^{\circ} \mathrm{C}$ (Dako, Agilent Technologies, Inc., Santa Clara, CA, USA) and observed under a light microscope (Leica Microsystems, Ltd., Milton Keynes, UK). Images were captured at a x200 magnification using Leica QWin Plus v3 software (Leica Microsystems, Ltd.). A graphic analysis system (Qianping Image Engineering Company, Wuhan, China) was used to semi-quantitate the density of RGMa. 

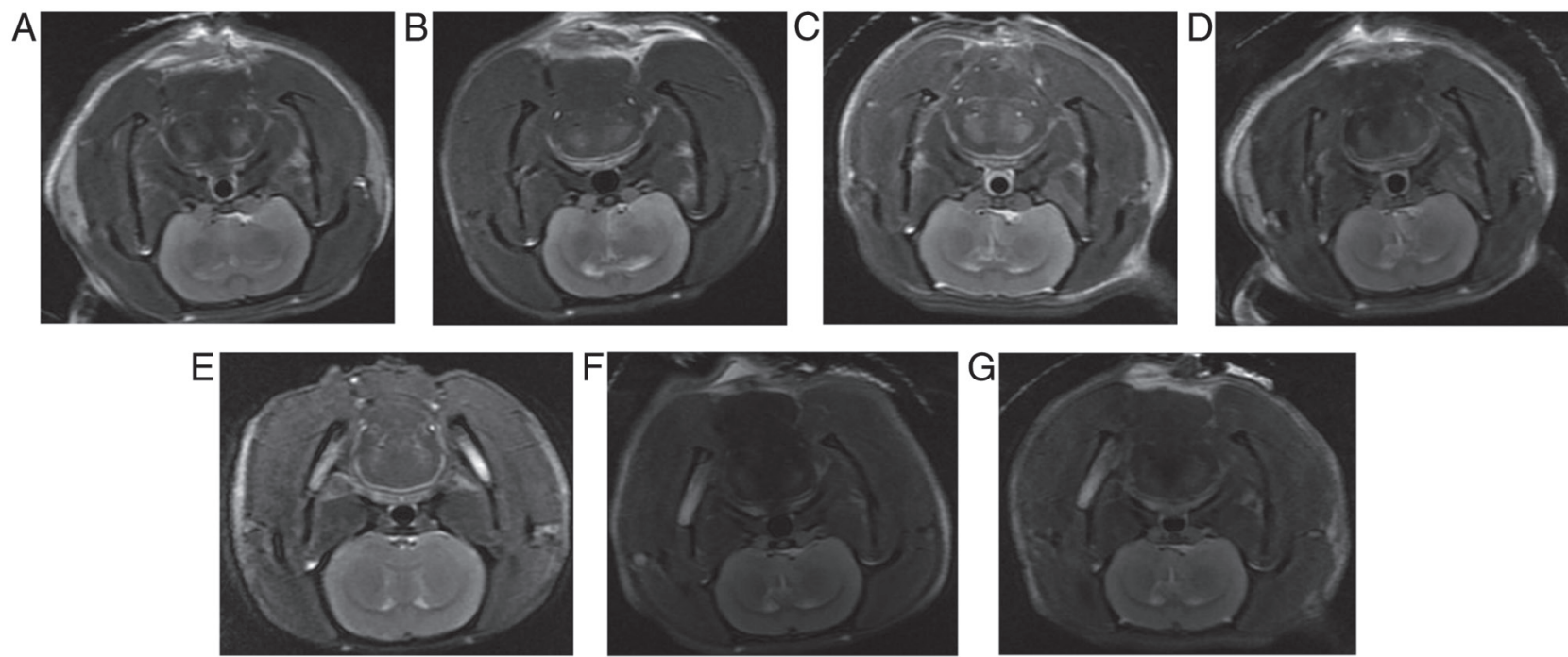

Figure 1. T2-weighted imaging of middle cerebral artery occlusion rats were obtained at (A) 1, (B) 3, (C) 6, (D) 9, (E) 12, (F) 24 and (G) $72 \mathrm{~h}$ following surgery.
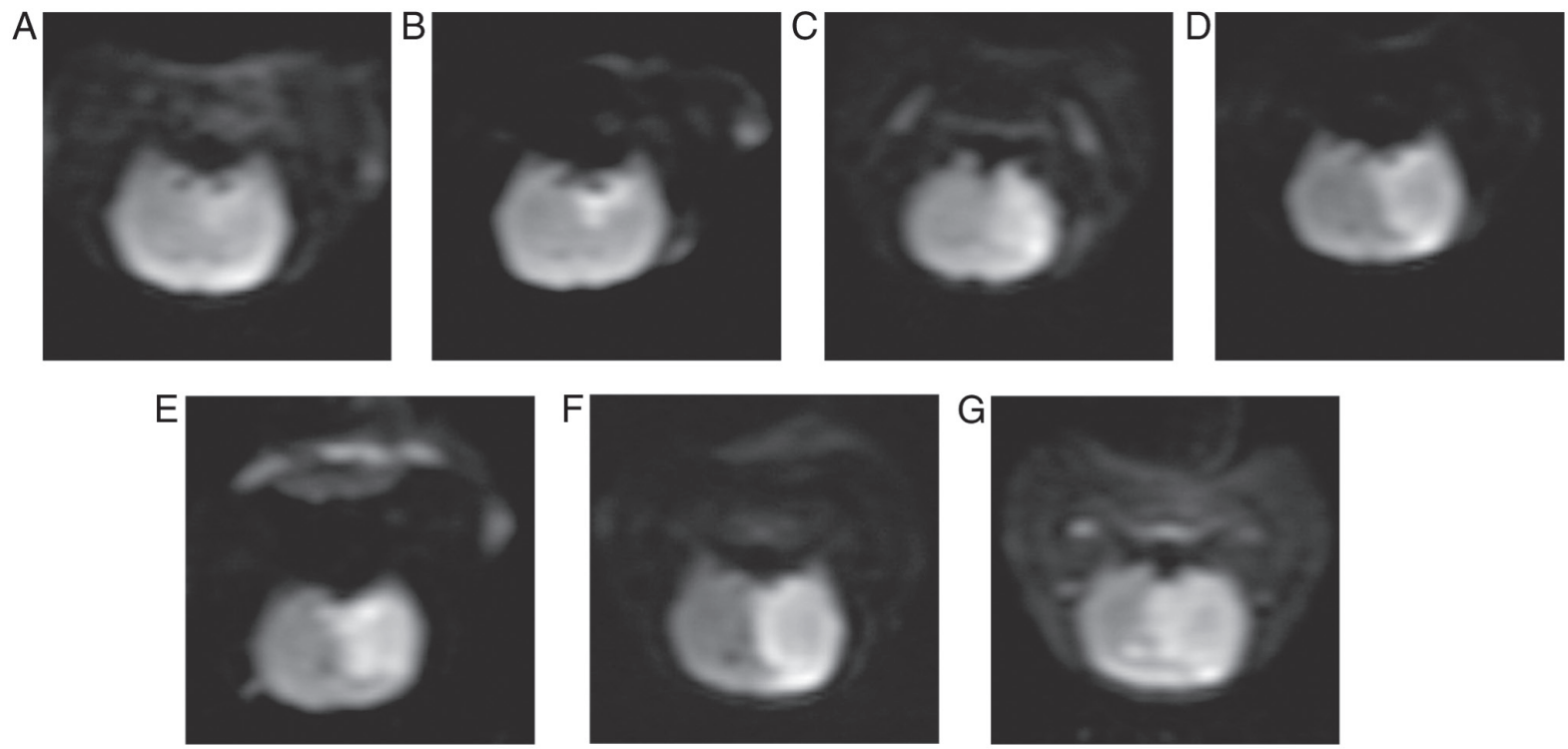

Figure 2. Diffusion-weighted imaging of middle cerebral artery occlusion rats were obtained at (A) 1, (B) 3, (C) 6, (D) 9, (E) 12, (F) 24 and (G) $72 \mathrm{~h}$ following surgery.

Statistical analysis. Data were analyzed using SAS software (SAS, 2002; SAS Institute, Inc., Cary, NC, USA). Data were presented as the mean \pm standard deviation. Data were analyzed using one-way analysis of variance followed by the least significant difference post-hoc test for multiple pairwise comparisons. The correlation between RMGa and FA was analyzed using Pearson's correlation. $\mathrm{P}<0.05$ was considered to indicate a statistically significant difference.

\section{Results}

T2WI and DWI image analysis of MCAO rats with bilateral cerebellar hemispheres. T2WI and DWI imaging of MCAO rats were obtained at 1, 3, 6, 9, 12, 24 and $72 \mathrm{~h}$ following surgery (Figs. 1 and 2). With the extension of infarct time, the infarct size expanded from the left basal ganglia to the entire left hemisphere and to the contralateral hemisphere. Notably, the infarct size was the largest at $12 \mathrm{~h}$ and gradually decreased thereafter.

FA values of MCAO rats with bilateral cerebellar hemispheres. At each time point, the ADC and FA values of the bilateral cerebellar hemispheres of MCAO rats were decreased compared with those of sham control rats (Tables I and II; and Fig. 3). This difference was maximal at $12 \mathrm{~h}$ following MCAO. Additionally, ADC was significantly decreased in contralateral (right) cerebellar hemisphere and the ipsilateral (left) cerebellar hemisphere at 9 and $12 \mathrm{~h}$ following MCAO compared with the sham control group. FA values were significantly decreased in contralateral (right) cerebellar hemisphere and the ipsilateral (left) cerebellar hemisphere compared with the sham control at 1, 3, 6, 9, 12 and $24 \mathrm{~h}$ following MCAO. 

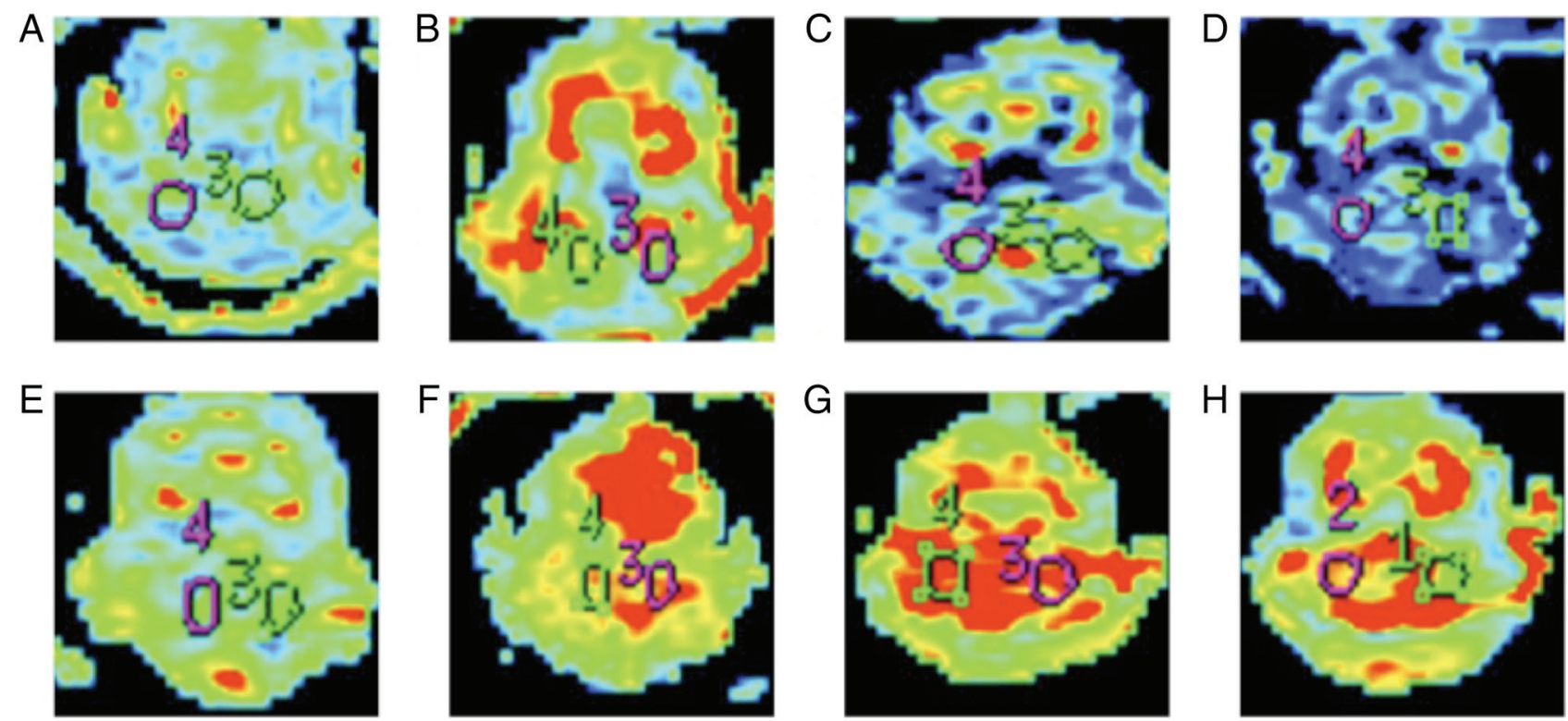

Figure 3. FA maps of the (A) sham control and middle cerebral artery occlusion rats in the bilateral cerebellar hemispheres at (B) 1, (C) 3, (D) 6, (E) 9, (F) 12, (G) 24 and $(\mathrm{H}) 72 \mathrm{~h}$ following infarction. The FA map of sham control rats represented the unaltered 1-72 $\mathrm{h}$ observations. Each of the other maps represented one of the three obtained images of the varied zones of infarction. Number 4 and the circle in the corresponding color represent signals from the left cerebellar hemisphere. Number 3 and the corresponding colored circles represent signals from the right cerebellar hemisphere. In figure $\mathrm{H}$, number 2 and the circle in the corresponding color represent signals from the left cerebellar hemisphere. Number 1 and the circle in the corresponding color represent signals from the right cerebellar hemisphere. FA, fractional anisotropy.
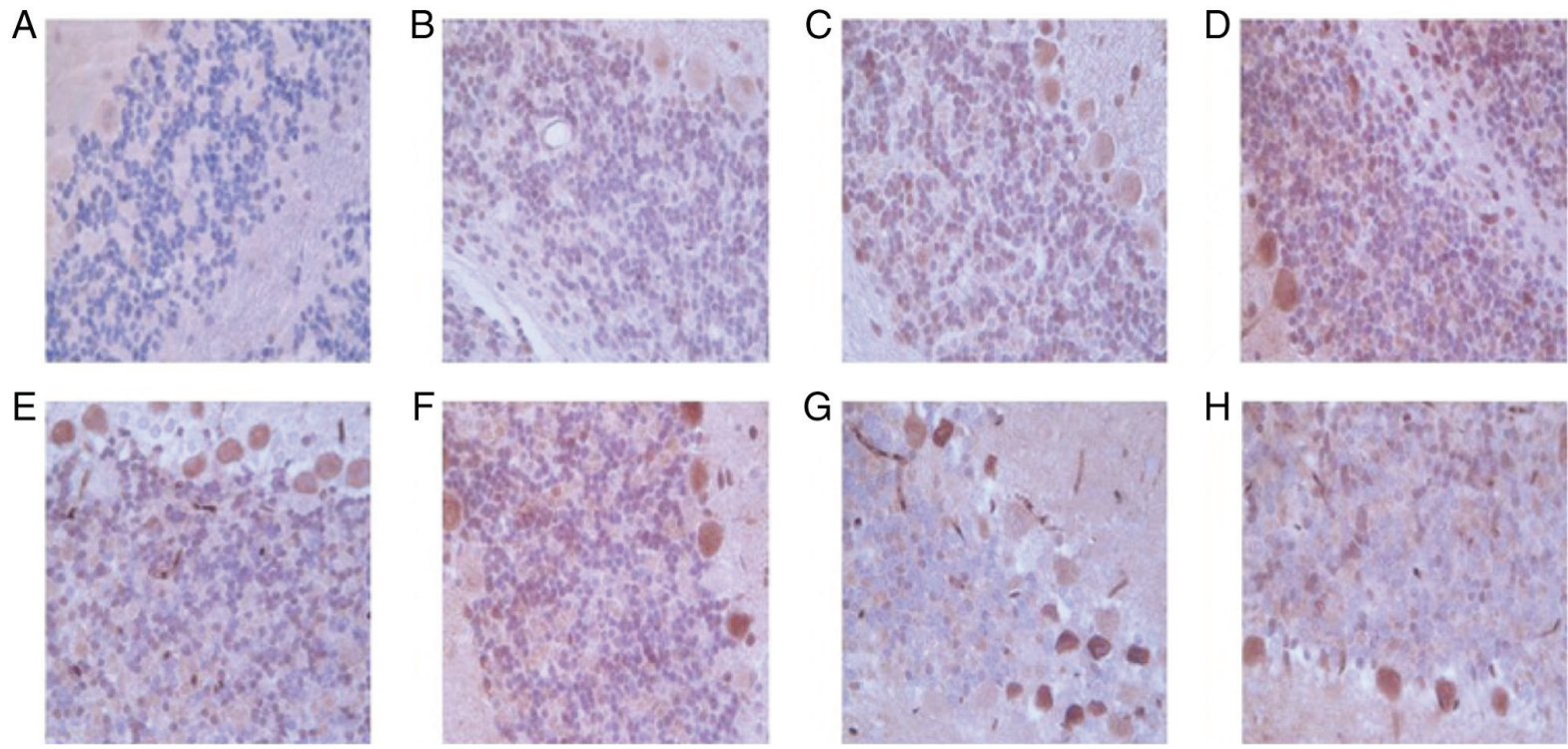

Figure 4. Representative images of immunohistochemical staining of RGMa in bilateral cerebellar hemispheres of the (A) sham control and MCAO rats at (B) 1, (C) 3, (D) 6, (E) 9, (F) 12, (G) 24 and (H) $72 \mathrm{~h}$ following infarction (magnification, x200). Integrated optical density value of the RGMa positive zone was used to semi-quantitatively measure the protein expression. (A) Representative image observed at $1-72 \mathrm{~h}$ of the infarction, whereas, each of the MCAO rat images were representative of three images from varied zones of infarction. MCAO, middle cerebral artery occlusion; RCMa, repulsive guidance molecule a.

The results suggested that the contralateral (right) cerebellar hemisphere indicated a large degree of decline compared with the ipsilateral (left) cerebellar hemisphere.

Protein expression of RMGa in bilateral cerebellar hemispheres of MCAO rats. RGMa protein expression level was increased over time, with maximal expression indicated at $24 \mathrm{~h}$ following infarction. RGMa protein expression was significantly increased in contralateral (right) cerebellar hemisphere and the ipsilateral (left) cerebellar hemisphere compared with sham control at 1, 3, 6, 9, 12, and $24 \mathrm{~h}$ following MCAO. Furthermore, the RGMa protein expression level in the contralateral (right) cerebellar hemisphere was higher compared with that in the ipsilateral (left) cerebellar hemisphere (Table III and Fig. 4). The results suggested that RGMa expression was the largest at $12 \mathrm{~h}$ and gradually decreased thereafter following MCAO in bilateral cerebellar hemispheres of rats, and RGMa expression in contralateral 
Table I. Apparent diffusion coefficient value of the sham control and MCAO groups at different time points.

\begin{tabular}{lccc}
\hline \multirow{2}{*}{ Group } & \multicolumn{2}{c}{ Cerebellum } & \\
\cline { 2 - 3 } & Time (h) & Right side & Light side \\
\hline Sham control & 0 & $6.95 \pm 0.83$ & $6.96 \pm 0.85$ \\
MCAO & 1 & $6.87 \pm 0.83$ & $6.81 \pm 1.31$ \\
& 3 & $6.84 \pm 0.92$ & $6.67 \pm 1.51$ \\
& 6 & $6.37 \pm 1.24$ & $6.52 \pm 1.83$ \\
& 9 & $6.05 \pm 1.08^{\mathrm{a}}$ & $6.27 \pm 0.86^{\mathrm{a}}$ \\
& 12 & $5.54 \pm 0.9^{\mathrm{a}}$ & $5.49 \pm 0.41^{\mathrm{a}}$ \\
& 24 & $6.36 \pm 0.32$ & $6.47 \pm 1.24$ \\
& 72 & $6.96 \pm 0.62$ & $6.57 \pm 1.24$ \\
\hline
\end{tabular}

${ }^{\mathrm{a}} \mathrm{P}<0.05$ vs. Sham control. MCAO, middle cerebral artery occlusion.

Table II. FA value of the control and MCAO group at different time points.

\begin{tabular}{lccc}
\hline \multirow{2}{*}{ Group } & \multicolumn{2}{c}{ Cerebellum } & \\
\cline { 2 - 3 } & Time $(\mathrm{h})$ & Right side & Light side \\
\hline Sham control & 0 & $0.299 \pm 0.033$ & $0.299 \pm 0.033$ \\
MCAO & 1 & $0.245 \pm 0.041^{\mathrm{a}}$ & $0.256 \pm 0.060$ \\
& 3 & $0.244 \pm 0.043^{\mathrm{a}}$ & $0.253 \pm 0.052^{\mathrm{a}}$ \\
& 6 & $0.237 \pm 0.064^{\mathrm{a}}$ & $0.251 \pm 0.021^{\mathrm{a}}$ \\
& 9 & $0.236 \pm 0.045^{\mathrm{a}}$ & $0.246 \pm 0.049^{\mathrm{a}}$ \\
& 12 & $0.228 \pm 0.042^{\mathrm{a}}$ & $0.244 \pm 0.033^{\mathrm{a}}$ \\
& 24 & $0.250 \pm 0.014^{\mathrm{a}}$ & $0.265 \pm 0.033$ \\
& 72 & $0.265 \pm 0.024$ & $0.269 \pm 0.034$ \\
\hline
\end{tabular}

${ }^{\mathrm{a}} \mathrm{P}<0.05$ vs. Sham control. MCAO, middle cerebral artery occlusion.

Table III. Expression of RGMa protein at different time points following MCAO.

\begin{tabular}{lccc}
\hline \multirow{2}{*}{ Group } & \multicolumn{2}{c}{ Cerebellum } & \\
\cline { 2 - 3 } & Time (h) & Right side & Light side \\
\hline Sham control & 0 & $83.06 \pm 2.87$ & $83.06 \pm 2.87$ \\
MCAO & 1 & $101.5 \pm 4.42^{\mathrm{a}}$ & $95.38 \pm 2.56^{\mathrm{a}}$ \\
& 3 & $111.05 \pm 5.57^{\mathrm{a}}$ & $100.98 \pm 4.32^{\mathrm{a}}$ \\
& 6 & $113.8 \pm 7.56^{\mathrm{a}}$ & $103.28 \pm 9.71^{\mathrm{a}}$ \\
& 9 & $118.52 \pm 9.59^{\mathrm{a}}$ & $103.71 \pm 6.73^{\mathrm{a}}$ \\
& 12 & $120.23 \pm 7.68^{\mathrm{a}}$ & $105.27 \pm 2.5^{\mathrm{a}}$ \\
& 24 & $136.44 \pm 5.10^{\mathrm{a}}$ & $120.74 \pm 5.06^{\mathrm{a}}$ \\
& 72 & $122.96 \pm 9.8^{\mathrm{a}}$ & $109.46 \pm 5.27^{\mathrm{a}}$
\end{tabular}

Signal of RGMa was semi-quantitated using graphic analysis software. The values were presented as the mean \pm standard deviation of three measures. ${ }^{\mathrm{a}} \mathrm{P}<0.05$ vs. Sham control. MCAO, middle cerebral artery occlusion; RGMa, RCMa, repulsive guidance molecule a.

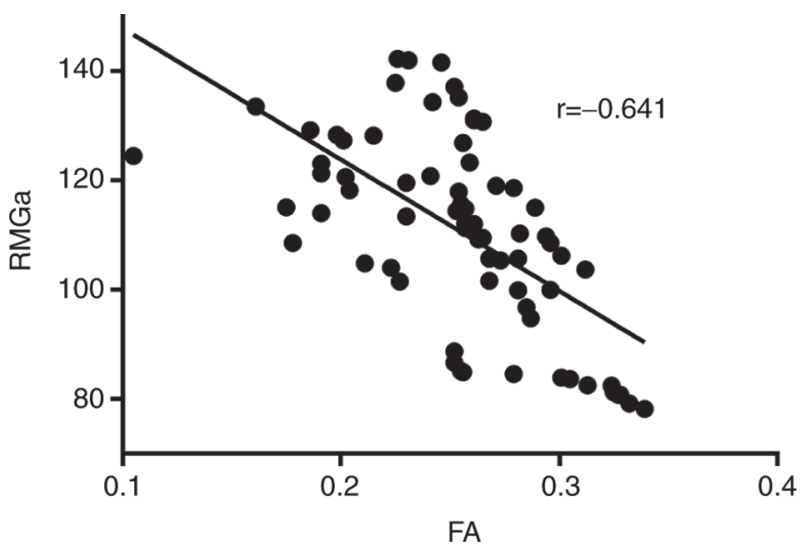

Figure 5. Correlation between FA changes and the expression of RGMa protein. RCMa, repulsive guidance molecule a; FA, fractional anisotropy.

(right) cerebellar hemisphere was higher than that in in the ipsilateral (left) cerebellar hemisphere.

Correlation between FA changes and expression of RGMa protein. To determine the association between the changes in FA and the expression of RGMa protein in the bilateral cerebellar hemispheres measured at 1, 3, 6, 9, 12, 24 and $72 \mathrm{~h}$ following MCAO, correlation analysis was performed. The results indicated that FA values and the expression of RGMa protein were negatively correlated (Spearman correlation coefficient $r=-0.641, \mathrm{P}<0.05$; Fig. 5).

\section{Discussion}

The objective of the present study was to use MR-DTI to detect the changes of distant regional diffusion parameters after supratentorial cerebral infarction in rats. A limited number of studies have assessed supratentorial cerebral infarction with MR-DTI. Notably, DTI based on DWI is a relatively novel technique that can reveal the direction of molecular diffusion (23-26). In the present study, DWI allowed for the examination of changes in the cerebellum located at a distance from the infracted site. Subsequently, RGMa, an inhibitor of axon growth, was used to investigate its role in CCD following supratentorial cerebral infarction.

Previously, DTI was mostly used to evaluate diffusion changes in the core area of acute infarction $(27,28)$. Only a few reports have evaluated changes in the regions remote from the supratentorial infarction, including the thalamus (29), pons (30) and cerebellum (9). The calculated FA values based on DTI reflect the extent of directional sensitivity of water diffusion, a measure of fiber connectivity within a voxel. Therefore, DWI serves as an appropriate measure of the possible alterations in microstructures of white matter tract (31). In the present study, the Longa's suture method with some modifications was used to establish the rat cerebral ischemia model of MCAO. Results indicated that a reduction in the FA values of the core region of the infarction and in the bilateral cerebellum was detectable at $1 \mathrm{~h}$ of cerebral infarction and continued to decline until $12 \mathrm{~h}$. Following the 12-h time point, the FA values began to recover; however, the FA values were still lower in the MCAO group compared with the sham 
control group. Perhaps due to the modified MCAO technique, a pre-eminent infarction was induced and therefore it was not completely recovered within $72 \mathrm{~h}$. The results confirmed the change in diffusion parameters in the regions remote from the infracted cerebellum. Furthermore, in the hyperacute and acute phases after cerebral ischemia in rats, a significant decrease in ADC values in the infarct core region was detected. It was previously proposed that, following a hyperacute supratentorial infarction, the blood flow volume in the brain microcirculation was reduced and caused dysfunctioning of the $\mathrm{Na}^{+}-\mathrm{K}^{+}$pump (32). As a result, cellular ionic homeostasis was disrupted, which accounted for movement of extracellular water into cells, thereby resulting in edema and cell death (33). These physiological changes affect the integrity of nerve fibers connecting the cerebral cortex to both sides of the brain, thereby impeding the transmission of neural signals sent by the cerebral cortex to both sides of the brain. Previous findings have suggested that the diffusion of water into the intracellular compartment is impeded by the presence of organelles in acute cerebellar infarction. Subsequently, the narrowing of the extracellular space due to the swelling of cells decreased the ADC values $(16,34)$. The FA measures in the white matter reflect fiber density, axonal diameter and myelination $(27,35)$. In the present study, the changes in FA in the bilateral cerebral hemispheres of the MCAO rats were significantly decreased compared with the sham control group. This observation may account for changes observed following supra-tentorial infarction, including changes the normal conduction of the corpus callosum combined with bilateral cerebral hemispheres and the cortical-pons-cerebellum path, ultimately resulting in limited function in distant regions, which may cause corresponding changes in blood flow and metabolic activity of coupled cerebellar regions (36-38). Thus, reduced FA values reflecting the atrophy of nerve fiber bundles affect the degree and direction of the diffusion of water molecules within the CCD-bridge-cerebellar cortex network.

In the present study, the reduced FA values of bilateral cerebellar hemispheres following the core area infarct suggested the occurrence of CCD following supratentorial cerebral infarction. The infarction-induced change in the contralateral cerebellar hemisphere was significantly greater than that of the ipsilateral cerebellar hemisphere. The possible cause of this phenomenon is that most nerve fibers connect the cerebral cortex to the contralateral cerebellum, whereas only a small number of these tracts relate to the ipsilateral cerebellum (39). Therefore, following infarction, the right cerebellar hemisphere was affected more predominantly compared with the left cerebellar hemisphere.

Supratentorial infarction caused atrophy of the neuronal fibers and functional depression in the local region of the lesion as well as in the distal site $(38,40)$. The severity and rate of necrosis of neuronal cells were affected by several factors $(16,41)$. Among them, RGMa was identified to inhibit axonal growth and neural tube closure, as well as sham control the proliferation and differentiation of neuronal cells via repulsive guidance signals that exist in the choroid plexus of normal rats, cerebellar Purkinje fibers and perivascular and brainstem neurons (42). Schwab et al (17) identified that RGMa was accumulated in scar tissue following spinal cord injury. Furthermore, the use of RGMa antibody reversed the axonal growth inhibitory effect of RGMa. As a result, neurological degeneration was blocked, thereby suggesting that RGMa participated in axonal growth inhibition after central nervous system injury. Recent in vitro functional studies using loss of function models have evidenced the direct involvement of specific guidance molecules in determining the growth and targeting of axonal fibers $(15,43)$. Thus, in the absence of these guidance molecules, the growing axons lose their directional sense and result in disturbances in the well-defined anatomical construction of axonal networks in the hippocampus (44). The inhibiting role of RGMa in neurite outgrowth and spinal cord injury has been demonstrated in vitro and in vivo (45-47); however, little is known regarding its contribution in cerebral ischemia. The present study indicated that RGMa protein expression was elevated after $1 \mathrm{~h}$ of the cerebral infarction and was maximal at $24 \mathrm{~h}$. However, a slight decrease in RGMa protein levels was revealed at $72 \mathrm{~h}$. Nevertheless, this level was higher in the tissue from the MCAO group compared with that in sham control group. These findings suggest that the elevated expression of RGMa protein in the core area of the supratentorial infarction and the remotely affected area of the cerebellum may be associated with axonal atrophy in these regions. Notably, the gain- and loss-of-function analysis of RGMa has demonstrated a role of this protein in controlling the proliferation and differentiation of neuronal cells and axon guidance (16).

In previous studies, in the early phase of acute cerebral ischemia, RGMa protein overexpression was demonstrated to inhibit the regeneration of nerve fibers and hindered the recovery of neurological function. The RGMa-mediated inhibition of axonal growth manifested as demyelination and atrophy of nerve fibers. These changes led to the defective diffusion of water molecules in brain tissue, which subsequently resulted in decreased FA values $(48,49)$. The decrease in FA values in the supratentorial infarction area and the affected remote region of bilateral cerebellar hemispheres was accompanied with an increased expression of RGMa protein in these regions, thereby indicating a negative correlation.

The present study had certain limitations. The mechanisms underlying RGMa-mediated regulation of FA and the development of CCD and MCAO have not been elucidated.

In conclusion, an established rat MCAO model of ischemia-induced neuronal injury indicated that reduced FA values were associated with increased expression of RGMa protein. These results also confirmed that MR-DTI is reasonably sensitive for detecting CCD, even as early as $1 \mathrm{~h}$ following the occurrence of a supratentorial infarction. This non-invasive technique is useful in measuring secondary changes in the distant regions after supratentorial infarction. Furthermore, the measure of RGMa protein expression reflected the extent of CCD in the present study and may be used in understanding the mechanisms of neuronal atrophy.

\section{Acknowledgements}

Not applicable.

\section{Funding}

No funding was received. 


\section{Availability of data and materials}

The datasets used and/or analyzed during the present study are available from the corresponding author on reasonable request.

\section{Authors' contributions}

YZ and YY conceived and designed the present study. YZ, $\mathrm{XW}$ and JC developed the methodology. YZ, XW, JC, YL and LY completed the experiments and collected the data. YZ, LY and $\mathrm{ZC}$ analyzed and interpreted the data. YZ and YY drafted the manuscript. All authors read and approved the final manuscript.

\section{Ethics approval and consent to participate}

The experimental protocols were approved by the Institutional Animal Care and Use Committee of Zhengzhou University (Zhengzhou, China).

\section{Patient consent for publication}

Not applicable.

\section{Competing interests}

The authors declare that there are no competing interests.

\section{References}

1. Mendis S: Stroke disability and rehabilitation of stroke: World Health Organization perspective. Int J Stroke 8: 3-4, 2013.

2. Savitz SI and Caplan LR: Vertebrobasilar disease. N Engl J Med 352: 2618-2626, 2005.

3. Gupta R, Joshi S, Mittal A, Luthra I, Mittal P and Verma V: Magnetic resonance imaging depiction of acquired Dyke-Davidoff-Masson syndrome with crossed cerebro-cerebellar diaschisis: Report of two cases. J Pediatr Neurosci 10: 294-296, 2015.

4. Kang KM, Sohn CH, Kim BS, Kim YI, Choi SH, Yun TJ, Kim JH, Park SW, Cheon GJ and Han MH: Correlation of asymmetry indices measured by arterial Spin-Labeling MR Imaging and SPECT in patients with crossed cerebellar diaschisis. AJNR Am J Neuroradiol 36: 1662-1668, 2015.

5. Komaba Y, Mishina M, Utsumi K, Katayama Y, Kobayashi S and Mori O: Crossed cerebellar diaschisis in patients with cortical infarction: Logistic regression analysis to control for confounding effects. Stroke 35: 472-476, 2004.

6. Kim J, Lee SK, Lee JD, Kim YW and Kim DI: Decreased fractional anisotropy of middle cerebellar peduncle in crossed cerebellar diaschisis: Diffusion-tensor imaging-positron-emission tomography correlation study. AJNR Am J Neuroradiol 26 : 2224-2228, 2005.

7. Dani KA, Santosh C, Brennan D, Hadley DM and Muir KW: Crossed cerebellar diaschisis: Insights into oxygen challenge MRI. J Cereb Blood Flow Metab 32: 2114-2117, 2012.

8. Baron JC, Bousser MG, Comar D and Castaigne P: 'Crossed cerebellar diaschisis' in human supratentorial brain infarction. Trans Am Neurol Assoc 105: 459-461, 1981.

9. Patay Z, Parra C, Hawk H, George A, Li Y, Scoggins M, Broniscer A and Ogg RJ: Quantitative longitudinal evaluation of diaschisis-related cerebellar perfusion and diffusion parameters in patients with supratentorial hemispheric high-grade gliomas after surgery. Cerebellum 13: 580-587, 2014.

10. Takasawa M, Hashikawa K, Ohtsuki T, Imaizumi M, Oku N, Kitagawa K, Hori $\mathrm{M}$ and Matsumoto M: Transient crossed cerebellar diaschisis following thalamic hemorrhage. J Neuroimaging 11: 438-440, 2001.
11. Strother MK, Buckingham C, Faraco CC, Arteaga DF, Lu P, $\mathrm{Xu} \mathrm{Y}$ and Donahue MJ: Crossed cerebellar diaschisis after stroke identified noninvasively with cerebral blood flow-weighted arterial spin labeling MRI. Eur J Radiol 85: 136-142, 2016.

12. Liu Y, Karonen JO, Nuutinen J, Vanninen E, Kuikka JT and Vanninen RL: Crossed cerebellar diaschisis in acute ischemic stroke: A study with serial SPECT and MRI. J Cereb Blood Flow Metab 27: 1724-1732, 2007.

13. Peled S and Yeshurun Y: Superresolution in MRI: Application to human white matter fiber tract visualization by diffusion tensor imaging. Magn Reson Med 45: 29-35, 2001.

14. Yoon B, Kim JS, Lee KS, Kim BS, Chung SR and Kim YI: Early pathological changes in the cerebellum of patients with pure cerebellar syndrome demonstrated by diffusion-tensor imaging. Eur Neurol 56: 166-171, 2006.

15. Hata K, Fujitani M, Yasuda Y, Doya H, Saito T, Yamagishi S, Mueller BK and Yamashita T: RGMa inhibition promotes axonal growth and recovery after spinal cord injury. J Cell Biol 173: 47-58, 2006.

16. Matsunaga E, Nakamura $\mathrm{H}$ and Chédotal A: Repulsive guidance molecule plays multiple roles in neuronal differentiation and axon guidance. J Neurosci 26: 6082-6088, 2006.

17. Schwab JM, Conrad S, Monnier PP, Julien S, Mueller BK and Schluesener HJ: Spinal cord injury-induced lesional expression of the repulsive guidance molecule (RGM). Eur J Neurosci 21: $1569-1576,2005$.

18. Key B and Lah GJ: Repulsive guidance molecule A (RGMa) A molecule for all seasons. Cell Adh Migr 6: 85-90, 2012

19. Severyn CJ, Shinde U and Rotwein P: Molecular biology, genetics and biochemistry of the repulsive guidance molecule family. Biochem J 422: 393-403, 2009.

20. Wang X, Cheng JL, Ran YC, Zhang Y, Yang L and Lin YN: Expression of RGMb in brain tissue of MCAO rats and its relationship with axonal regeneration. J Neurol Sci 383: 79-86, 2017.

21. Wang Y, Zhang R, Xing X, Guo J, Xie F, Zhang G and Qin X: Repulsive guidance molecule asuppresses angiogenesis after ischemia/reperfusion injury of middle cerebral artery occlusion in rats. Neurosci Lett 662: 318-323, 2018.

22. Longa EZ, Weinstein PR, Carlson S and Cummins R: Reversible middle cerebral artery occlusion without craniectomy in rats. Stroke 20: 84-91, 1989.

23. Zheng G, Chen X, Xu B, Zhang J, Lv X, Li J, Li F, Hu S, Zhang $\mathrm{T}$ and $\mathrm{Li}$ Y: Plasticity of language pathways in patients with low-grade glioma: A diffusion tensor imaging study. Neural Regen Res 8: 647-654, 2013.

24. Kwon YH, Jang SH and Yeo SS: Age-related changes of lateral ventricular width and periventricular white matter in the human brain: A diffusion tensor imaging study. Neural Regen Res 9: 986-989, 2014

25. Meng X, Wang Q, Hou J, Zhang X, Wang E, Li Q, Zeng Q, Wang Q, $\mathrm{Li} \mathrm{C}$ and Ma X: Diffusion tensor imaging of normal-appearing white matter in unilateral cerebral arterial occlusive disease. J Magn Reson Imaging 38: 650-654, 2013.

26. Zhang Y, Wan S and Zhang X: Geniculocalcarine tract disintegration after ischemic stroke: A diffusion tensor imaging study. AJNR Am J Neuroradiol 34: 1890-1894, 2013.

27. Le Bihan D, Mangin JF, Poupon C, Clark CA, Pappata S, Molko $\mathrm{N}$ and Chabriat H: Diffusion tensor imaging: Concepts and applications. J Magn Reson Imaging 13: 534-546, 2001.

28. Zhao J, Chang W and Liu Y: Clinical application of diffusion tensor imaging in acute stroke. Clin Med China 30: 1166-1168, 2014.

29. Erbetta A, Mandelli M, Savoiardo M, Grisoli M, Bizzi A, Soliveri P, Chiapparini L, Prioni S, Bruzzone MG and Girotti F: Diffusion tensor imaging shows different topographic involvement of the thalamus in progressive supranuclear palsy and corticobasal degeneration. AJNR Am J Neuroradiol 30: 1482-1487, 2009

30. Karampinos DC, Van AT, Olivero WC, Georgiadis JG and Sutton BP: High-resolution diffusion tensor imaging of the human pons with a reduced field-of-view, multishot, variable-density, spiral acquisition at 3 T. Magn Reson Med 62: 1007-1016, 2009.

31. Lao Y, Kang Y, Collignon O, Brun C, Kheibai SB, Alary F, Gee J, Nelson MD, Lepore F and Lepore N: A study of brain white matter plasticity in early blinds using tract-based spatial statistics and tract statistical analysis. Neuroreport 26: 1151-1154, 2015.

32. Brillault J, Lam TI, Rutkowsky JM, Foroutan S and O'Donnell ME: Hypoxia effects on cell volume and ion uptake of cerebral microvascular endothelial cells. Am J Physiol Cell Physiol 294: C88-C96, 2008. 
33. Yang GY, Chen SF, Kinouchi H, Chan PH and Weinstein PR Edema, cation content, and ATPase activity after middle cerebral artery occlusion in rats. Stroke 23: 1331-1336, 1992.

34. Sorensen AG, Wu O, Copen WA, Davis TL, Gonzalez RG Koroshetz WJ, Reese TG, Rosen BR, Wedeen VJ and Weisskoff RM: Human acute cerebral ischemia: Detection of changes in water diffusion anisotropy by using MR imaging. Radiology 212: 785-792, 1999.

35. Foong J, Maier M, Clark CA, Barker GJ, Miller DH and Ron MA: Neuropathological abnormalities of the corpus callosum in schizophrenia: A diffusion tensor imaging study. J Neurol Neurosurg Psychiatry 68: 242-244, 2000.

36. Ito H, Takahashi K, Hatazawa J, Kim SG and Kanno I: Changes in human regional cerebral blood flow and cerebral blood volume during visual stimulation measured by positron emission tomography. J Cereb Blood Flow Metab 21: 608-612, 2001.

37. Devor A, Ulbert I, Dunn AK, Narayanan SN, Jones SR, Andermann ML, Boas DA and Dale AM: Coupling of the cortical hemodynamic response to cortical and thalamic neuronal activity. Proc Natl Acad Sci USA 102: 3822-3827, 2005.

38. Ishihara M, Kumita S, Mizumura S and Kumazaki T: Crossed cerebellar diaschisis: The role of motor and premotor areas in functional connections. J Neuroimaging 9: 30-33, 1999.

39. Jeon YW, Kim SH, Lee JY, Whang K, Kim MS, Kim YJ, and Lee MS: Dynamic CT perfusion imaging for the detection of crossed cerebellar diaschisis in acute ischemic stroke. Korean J Radiol 13:12-9, 2012.

40. Kim SE and Lee MC: Cerebellar vasoreactivity in stroke patients with crossed cerebellar diaschisis assessRed by acetazolamide and 99mTc-HMPAO SPECT. J Nucl Med 41: 416-420, 2000

41. Matsunaga E, Tauszig-Delamasure S, Monnier PP, Mueller BK, Strittmatter SM, Mehlen P and Chédotal A: RGM and its receptor neogenin regulate neuronal survival. Nat Cell Biol 6: 749-755, 2004 .
42. Tian C and Liu J: Repulsive guidance molecules (RGMs) and neogenin in bone morphogenetic protein (BMP) signaling. Mol Reprod Dev 80: 700-717, 2013.

43. Wang T, Wu X, Yin C, Klebe D, Zhang JH and Qin X: CRMP-2 is involved in axon growth inhibition induced by RGMa in vitro and in vivo. Mol Neurobiol 47: 903-913, 2013.

44. Brinks H, Conrad S, Vogt J, Oldecamp J, Sierra A, Deitinghoff L, Bechmann I, Alvarez-Bolado G, Heimrich B, Monnier PP et al: The repulsive guidance molecule RGMa is involved in the formation of afferent connections in the dentate gyrus. J Neurosci 24: 3862-3869, 2004.

45. Metzger M, Conrad S, Skutella T and Just L: RGMa inhibits neurite outgrowth of neuronal progenitors from murine enteric nervous system via the neogenin receptor in vitro. J Neurochem 103: 2665-2678, 2007.

46. Tassew NG, Charish J, Seidah NG and Monnier PP: SKI-1 and Furin generate multiple RGMa fragments that regulate axonal growth. Dev Cell 22: 391-402, 2012.

47. Tao T, Xu G, Si Chen C, Feng J, Kong Y and Qin X: Minocycline promotes axonal regeneration through suppression of RGMa in rat MCAO/reperfusion model. Synapse 67: 189-198, 2013.

48. Feng J, Wang T, Li Q, Wu X and Qin X: RNA interference against repulsive guidance molecule $\mathrm{A}$ improves axon sprout and neural function recovery of rats after MCAO/reperfusion. Exp Neurol 238: 235-242, 2012.

49. Nimsky C, Ganslandt O, Hastreiter P, Wang R, Benner T, Sorensen AG and Fahlbusch R: Intraoperative diffusion-tensor MR imaging: Shifting of white matter tracts during neurosurgical procedures-initial experience. Radiology 234: 218-225, 2005. 